\title{
Localization of Human Platelet Autoantigens to the Cysteine-rich Region of Glycoprotein Illa
}

\author{
Riitta Kekomaki, * Barbara Dawson, ${ }^{\star}$ Janice McFarland, ${ }^{\star *}$ and Thomas J. Kunicki"* \\ *The Blood Center of Southeastern Wisconsin, Milwaukee, Wisconsin 53233; the ${ }^{\ddagger}$ Departments of Medicine and Pathology and \\ 'Departments of Anatomy/Cell Biology of the Medical College of Wisconsin, Milwaukee, Wisconsin 53226
}

\begin{abstract}
The object of this study was to further localize autoantigenic structures on IIb-IIIa and, if possible, to precisely identify the epitopes recognized by human autoantibodies. In this paper, we identify a 50-kD chymotryptic fragment of IIIa that is recognized by a high percentage of human autoantibodies, typified by the prototype IgG autoantibody RA, which binds to IIIa on intact platelets as well as in an immunoblot assay under nonreduced conditions. Using an immunoblot assay, a carboxy-terminal region of this fragment $(33 \mathrm{kD})$ that contains the cysteine-rich domains of IIIa was found to carry the epitope(s) recognized by the prototype autoantibody RA. The amino-terminal amino acid sequence of the reduced 33-kD fragment, the smallest fragment that retains the $R A$ epitope, is XPSQQDEXSP, and that of the reduced 50-kD fragment is IVQVTFD. This indicates that the 33-kD fragment consists of $\sim 175$ amino acids beginning at residue 479 and extending at least through residues $636-654$, while the 50-kD fragment spans the same region but begins at residue 427 . It is apparent that the 33-kD fragment is generated from the $50-\mathrm{kD}$ fragment by additional chymotryptic hydrolysis but remains associated because of the multiple disulfide bonds that are characteristic of this cysteine-rich domain. Sera from $48 \%$ of patients with chronic ITP and 2 of 8 patients with acute ITP contain antibodies that bind to the 50-kD fragment in an ELISA. Antibodies of the same specificity are also found in one-third of patients with either secondary immune thrombocytopenia or apparent nonimmune thrombocytopenia. We conclude that the 50-kD cysteine-rich region of IIIa is a frequent target of autoantibodies in ITP, but that such antibodies may also be present in cases of thrombocytopenia that cannot be linked to an apparent autoimmune process. (J. Clin. Invest. 1991. 88:847-854.) Key words: platelet • glycoprotein IIIa • autoantigen • thrombocytopenia
\end{abstract}

\section{Introduction}

The glycoprotein IIb-IIIa complex of human platelets is known to carry a number of clinically important alloantigens and autoantigens. This conclusion was initially based upon comparisons of platelets from normal subjects and patients with Glanz-

Dr. Thomas J. Kunicki, The Blood Center of Southeastern Wisconsin, 1701 West Wisconsin Ave., Milwaukee, WI 53233. Dr. Kekomaki's current address is Finnish Red Cross, Blood Transfusion Service, Kivihaantle 7, SF-00310 Helsinki, Finland.

Received for publication 1 March 1990 and in revised form 29 April 1991

J. Clin. Invest.

(c) The American Society for Clinical Investigation, Inc.

0021-9738/91/09/0847/08 \$2.00

Volume 88, September 1991, 847-854 mann's thrombasthenia, which are deficient in this glycoprotein complex $(1,2)$. The first indication that the $\mathrm{Pl}^{\mathrm{A}}$ alloantigen system is located on this glycoprotein complex was derived in studies of platelets from Glanzmann's thrombasthenia patients (3). Subsequent studies have confirmed this finding and have localized two other alloantigen systems, Bak and Pen, to IIb and IIIa, respectively (4-9).

The first report suggesting a preponderance of autoantibodies against IIb-IIIa showed that thrombasthenic platelets fail to react with autoantibodies from a subgroup of patients with autoimmune thrombocytopenia (10). In an immunoblot assay, Beardsley et al. were able to specifically identify IIIa as a target antigen in a majority of patients with chronic autoimmune thrombocytopenia (11). Autoantibodies specific for IIb have also been identified in at least two patients (12).

The importance of IIb-IIIa as a carrier of autoantigens in both chronic and acute ITP has been firmly established using novel MAb-based ELISA and RIAs (13-16). Based on these reports, as many as one-half of patients with chronic idiopathic (autoimmune) thrombocytopenic purpura (ITP) ${ }^{1}$ have circulating autoantibodies specific for the IIb-IIIa complex.

In this study, we further localize a region of IIIa that contains antigens recognized by a high percentage of autoantibodies from patients with ITP.

\section{Methods}

Blood samples. Blood samples from 131 patients with suspected idiopathic (autoimmune) thrombocytopenia, as determined by the referring physicians, were included in this study. All patients also had laboratory findings of elevated direct platelet-associated IgG and/or IgM, by nephelometry (17), as well as serum antibodies reactive with intact platelets, by ELISA (9). All patients were suspected of having autoimmune thrombocytopenia alone or in combination with other causes of thrombocytopenia. In 70 of these 131 patients, clinical histories were available (see Table I) and revealed that in 39 patients (54\%) ITP (either acute or chronic) was felt to be the primary cause of the thrombocytopenia. In 31 patients (46\%) other causes of thrombocytopenia were apparent, including secondary ITP (6 patients), drug-dependent immune thrombocytopenia ( 7 patients), and nonimmune thrombocytopenia (18 patients).

Antibodies. To identify antibodies that also react with denatured platelet antigens, plasma samples were analyzed in an immunoblot assay using total platelet protein as the target. Several antisera reactive with IIIa were identified in this manner, including RA and SD. Each of these antisera react only with nonreduced IIIa, a finding consistent with the original observation of Beardsley et al. (11). Since RA antiserum contains exceptionally high levels of antibody, it was used as the prototype for further studies of the epitopes recognized by this subset of autoantibodies. Anti-PI ${ }^{\mathrm{Al}}$ antisera were used as a positive control for the binding of human antibodies. The murine MAbs AP2 (anti-IIb-IIIa

1. Abbreviations used in this paper: ACE, antigen-capture ELISA; ITP, idiopathic thrombocytopenic purpura; MACE, modified ACE; PRP, platelet-rich plasma. 
complex) (18), AP4 (anti-IIb heavy chain) and AP5 (anti-IIIa) were used as specific markers of the respective glycoproteins. AP4 and AP5 bind specifically to IIb and IIIa, respectively, in an immunoblot assay of nonreduced and denatured platelet proteins (see Fig. 1). Murine IgG antibodies were purified from ascites fluid by HPLC using ABX columns (Whatman, Inc., Hillsboro, OR). Rabbit polyclonal antisera specific for glycoproteins IIb and IIIa were a gift from Dr. D. Pidard (U.150 INSERM, Paris, France). The sequence of glycoprotein IIIa has been determined (19), and antibodies specific for individual peptide sequences of IIIa have been developed. Rabbit polyclonal antibody specific for the IIIa amino-terminal sequence 1-13 (ab1) was a gift from Drs. J. Beer and B. Coller (Stony Brook, NY). Rabbit polyclonal antibody against another peptide derived from the sequence of III a (amino acids 636-654) was generously supplied by Drs. S. D'Souza and E. Plow (Scripps Research Foundation, La Jolla, CA).

Biotinylation of antibodies. $1 \mathrm{ml}$ of purified murine $\mathrm{IgG}(0.5 \mathrm{mg} /$

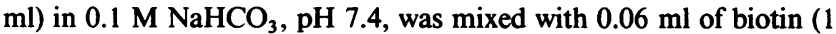
$\mathrm{mg} / \mathrm{ml}$; NHS-LC Biotin; Pierce Chemical Co., Rockford, IL) and incubated for $4 \mathrm{~h}$ at ambient temperature. The reaction was stopped by dialyzing the mixture against PBS, pH 7.4, containing $0.1 \% \mathrm{NaN}_{3}$ at $4^{\circ} \mathrm{C}$ overnight.

Isolation of platelets. $6 \mathrm{vol}$ of blood were mixed with 1 vol acid-citrate-dextrose solution (ACD; NIH formula A) and centrifuged at $250 \mathrm{~g}$ for $10 \mathrm{~min}$ at ambient temperature to obtain platelet-rich plasma (PRP). Prostaglandin $\mathrm{E}_{1}\left(\mathrm{PGE}_{1}\right.$; Sigma Chemical Co., St. Louis, MO) was added to the PRP to a final concentration of $20 \mathrm{ng} / \mathrm{ml}$. After a 15-min incubation, the PRP was centrifuged at $750 \mathrm{~g}$ for $10 \mathrm{~min}$ to sediment platelets. After three washes with Ringer's citrate-dextrose (RCD), pH 6.5 (20), the platelet pellet was resuspended in an appropriate buffer, and the platelet concentration determined using a Coulter Counter (Coulter Electronics, Hialeah, FL).

Antigen-capture ELISA (ACE). The ACE was performed as described by Furihata et al. (9) using solid-phase AP2 to capture IIb-IIIa or solid phase AP5 to capture the 50-kD chymotryptic fragment of IIIa (see below).

Modified antigen-capture ELISA (MACE). $50 \mu \mathrm{l}$ of purified murine monoclonal AP5 IgG $(10 \mu \mathrm{g} / \mathrm{ml})$ in $0.05 \mathrm{M}$ sodium carbonate buffer, pH 9.6, were added to each well of a flat-bottomed 96-well microtiter plate (Immunolon II; Dynatech Laboratories, Inc., Alexandria, VA) and incubated at $4^{\circ} \mathrm{C}$ overnight. The wells were then blotted dry and incubated with $67 \mathrm{mM} \mathrm{Na}_{2} \mathrm{PO}_{4}, 67 \mathrm{mM} \mathrm{NaHPO}, 150 \mathrm{mM} \mathrm{NaCl}, \mathrm{pH}$ 7.4, containing $0.05 \%$ (vol/vol) Tween 20 (PBS-Tween) and $1 \%$ (wt/ vol) gelatin at ambient temperature for 30 min to block the remaining binding sites. Washed platelets were sensitized by incubating $4 \times 10^{7}$

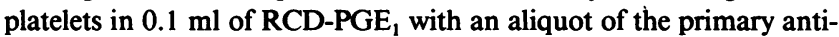
body (100-400 $\mu$ l of antisera or 1-6 $\mu \mathrm{g}$ of purified murine antibody) for $1 \mathrm{~h}$ at ambient temperature. The platelets were then washed three times with RCD-PGE ${ }_{1}$ and lysed in $40 \mathrm{mM}$ Tris, $145 \mathrm{mM}$ saline containing $1 \%$ (vol/vol) Triton X-100. Lysates were incubated at $4^{\circ} \mathrm{C}$ overnight with agitation and then centrifuged at $12,000 \mathrm{~g}$ for $5 \mathrm{~min}$. The supernatants were then diluted with an equal volume of PBS-Tween. $50 \mu \mathrm{l}$ of the diluted lysates were added to the wells of an AP5-coated microtiter plate, and the plate was incubated for $1 \mathrm{~h}$ at ambient temperature. The wells were washed three times with PBS-Tween, then $50 \mu$ l of biotinylated HB-43 (murine monoclonal anti-human IgG Fc; American Tissue Type Culture, Gaithersburg, MD) at a concentration of $1 \mu \mathrm{g} / \mathrm{ml}$ were added. The microtiter plates were incubated for $1 \mathrm{~h}$, and after three subsequent washes with PBS-Tween, $50 \mu \mathrm{l}$ of alkaline phosphatase-conjugated streptavidin (Zymed Laboratories, Inc., San Francisco, CA) diluted 1:1,500 were added to the wells. After 15 min, the wells were washed five more times with PBS-Tween, and the substrate (p-nitrophenylphosphate) in $100 \mathrm{mM}$ Tris, $100 \mathrm{mM} \mathrm{NaCl}, 5 \mathrm{mM}$ $\mathrm{MgCl}_{2}, \mathrm{pH} 9.5$, was added. The plates were incubated for $30 \mathrm{~min}$ at ambient temperature, and the absorbance at $405 \mathrm{~nm}$ was recorded using an automated microplate reader (EIA reader, Model 255, BioRad Laboratories, Mountain View, CA).

Direct antigen ELISA. In selected experiments, $50 \mu \mathrm{l}$ of purified IIb-IIla $(1 \mu \mathrm{g} / \mathrm{ml})$ or affinity-purified $50-\mathrm{kD}$ chymotryptic fragment of
IIIa were used to coat the microtiter wells. After blocking the wells with PBS-Tween containing $2 \%$ BSA, $50 \mu \mathrm{l}$ of diluted antiserum (primary antibody) were added to each well. After incubation at ambient temperature for $1 \mathrm{~h}$, washing and detection of bound human antibodies were performed as described above. Instead of biotinylated HB-43, AP-conjugated goat anti-human $I g(G+M+A)$ was used as the secondary antibody.

Proteolysis of platelets. All proteases were purchased from Worthington Biochemical Corp. (Freehold, NJ) and were of highest possible purity. Washed platelets were suspended in $20 \mathrm{mM}$ Hepes, $150 \mathrm{mM}$

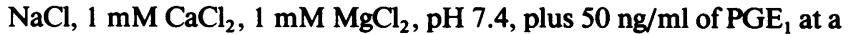
concentration of $1,000 \times 10^{9}$ platelets/liter. Chymotrypsin ( $\alpha$-chymotrypsin from bovine pancreas; $63 \mathrm{U} / \mathrm{mg}$ ) digestion was performed at $37^{\circ} \mathrm{C}$ for $30 \mathrm{~min}$ at a final protease concentration of $0.35 \mathrm{mg} / \mathrm{ml}$. For papain treatment, washed platelets were suspended in $20 \mathrm{mM}$ Hepes, $150 \mathrm{mM} \mathrm{NaCl}, 5 \mathrm{mM}$ EDTA, pH 7.4, plus $50 \mathrm{ng} / \mathrm{ml}$ of PGE $_{1}$. Papain (final concentration $100 \mu \mathrm{g} / \mathrm{ml}$ ) was preactivated by incubation in the presence of $5 \mathrm{mM}$ cysteine at $37^{\circ} \mathrm{C}$ for $30 \mathrm{~min}$ according to the instructions from the manufacturer. Thrombin (human $\alpha$-thrombin; Bureau of Biologicals, FDA, Bethesda, MD) activation of platelets was performed by incubating $10^{9}$ platelets in Hepes $\mathrm{NaCl}, 1 \mathrm{mM}$ EDTA, $\mathrm{pH}$ 7.4, plus $50 \mathrm{ng} / \mathrm{ml} \mathrm{PGE}_{1}$ with $0.1 \mathrm{U} / \mathrm{ml}$ of thrombin at $37^{\circ} \mathrm{C}$ for $5 \mathrm{~min}$. The reaction was stopped by adding hirudin (Sigma Chemical Co.) at a final concentration of $1 \mathrm{U} / \mathrm{ml}$. The platelets were washed twice with RCD-PGE 1 and either fixed with paraformaldehyde for flow cytometry (as described above) or suspended in Tris-glycine buffer for detergent lysis (as described below).

Solubilization of platelets in Triton X-100. Platelet lysates were prepared as described previously by Furihata et al. (21). In brief, platelets were suspended in 9 vol of $33 \mathrm{mM}$ Tris, $100 \mathrm{mM}$ glycine, $\mathrm{pH} \mathrm{8.7,} \mathrm{at} \mathrm{a}$ concentration of $5,000 \times 10^{9} /$ liter and mixed with $1 \mathrm{vol}$ of $10 \%$ (vol/ vol) Triton $\mathrm{X}-100$ in the same buffer. Lysates were centrifuged at $80,000 \mathrm{~g}$ for $30 \mathrm{~min}$ at $4^{\circ} \mathrm{C}$. The protein concentration of the supernatant was determined using the method of Markwell et al. (22).

Purification of platelet IIb-IIIa. The IIb-IIIa complex was isolated as described by Fitzgerald et al. (23). Platelets from $50 \mathrm{U}$ of outdated platelet concentrates served as the source of IIb-IIIa and were lysed in Triton X-100 as described above. Con A-Sepharose was used to adsorb a IIb-IIIa-enriched fraction that was eluted from the column with $\alpha$ methyl-D-mannose. The major contaminants, thrombospondin and fibrinogen, were then removed by passing the material over a heparinSepharose column and subsequent gel filtration with Sephacryl S-300. Trace amounts of fibrinogen still contaminating the preparation were removed by wheat germ agglutinin affinity chromatography. The purity of the final preparation was evaluated by SDS-PAGE and ELISA using murine MAbs specific for platelet glycoproteins.

Proteolysis of purified IIb-IIIa. The protein concentration of purified IIb-IIla was adjusted to $0.64 \mathrm{mg} / \mathrm{ml}$. The amount of the different proteases used is expressed as the ratio of the weight of the protease to the weight of IIb-IIIa. Chymotrypsin digestion was performed at $37^{\circ} \mathrm{C}$ for 16-20 h with an enzyme to protein ratio of $1: 7$ in $20 \mathrm{mM}$ Tris, 100 $\mathrm{mM} \mathrm{NaCl}, 3 \mathrm{mM} \mathrm{CaCl}_{2}, \mathrm{pH}$ 7.8. To terminate digestion, PMSF was added to a final concentration of $1 \mathrm{mM}$.

Immunoblot assay. Detection of immunoreactive proteolytic fragments of IIb-IIIa was performed by an immunoblot assay. SDS-PAGE was performed as described (24) using a Laemmli buffer system and 7 , $10,12.5$, or $15 \%$ polyacrylamide resolving slab gels. Proteins were then transferred electrophoretically to $0.2 \mu \mathrm{m}$ pore-size nitrocellulose (BioRad Laboratories) or to polyvinylidine difluoride (PVDF, Immobilon P; Millipore, Bedford, MA) membranes. In general, the nitrocellulose or PVDF membrane and the gel were first equilibrated in transfer buffer (192 mM glycine, $25 \mathrm{mM}$ M Tris, $20 \%$ methanol, pH 8.3). However, in selected experiments, proteins were first reduced in the polyacrylamide gel by equilibrating the entire slab gel in transfer buffer containing 5\% 2-mercaptoethanol for $\mathbf{4 5} \mathrm{min}$ at ambient temperature. Blotting was carried out at $30 \mathrm{~V}$ for $30 \mathrm{~min}$ and then at $60 \mathrm{~V}$ for $3 \mathrm{~h}$ $\left(4^{\circ} \mathrm{C}\right)$. After blotting, the remaining reactive sites of the nitrocellulose or PVDF membrane were blocked by incubating the sheets in PBS- 
Tween containing $0.05 \% \mathrm{NaN}_{3}$. If the membranes were not to be stained immediately, they were allowed to air-dry and stored in airtight plastic bags between two filter papers (No. 1; Whatman, Inc.) at $-20^{\circ} \mathrm{C}$. The membrane was then washed three times for $10 \mathrm{~min}$ in PBS-Tween. The same buffer containing $0.5 \%$ FCS was used to dilute either the antisera or purified antibodies. The membrane strips were incubated with the primary antibody overnight (16-20 h) at ambient temperature on a shaker, washed three times for $10 \mathrm{~min}$ and incubated with a 1:1,000 dilution of alkaline phosphatase-conjugated goat antibodies against human, rabbit, or mouse IgG (Zymed Laboratories, Inc.) or with a 1:1,500 dilution of alkaline phosphatase-conjugated streptavidin (when biotinylated primary antibodies were used). After 3 $h$, the strips were washed an additional three times and then incubated with a freshly prepared substrate solution. The substrate solution consisted of $66 \mu$ l of a stock solution of nitroblue tetrazolium (NBT; 50 $\mathrm{mg} / \mathrm{ml}$ in $70 \%$ dimethylformamide) and $33 \mu \mathrm{l}$ of a stock solution bromo-4-chloro-3-indolylphosphate (BCIP; $50 \mathrm{mg} / \mathrm{ml}$ in $100 \%$ dimethylformamide) in $10 \mathrm{ml}$ of $100 \mathrm{mM}$ Tris- $\mathrm{HCl}, 100 \mathrm{mM} \mathrm{NaCl}, 5$ $\mathrm{mM} \mathrm{MgCl} 2, \mathrm{pH}$ 9.5. To stop color development, the membranes were rinsed in distilled water for $10 \mathrm{~min}$ and allowed to dry.

Immunoaffinity purification of the core chymotryptic fragment of IIIa. Immobilized streptavidin (Pierce Chemical Co.) was used to capture biotinylated murine MAb AP5. Biotinylated AP5 was mixed with streptavidin-agarose ( $1 \mu \mathrm{g} \mathrm{AP5}$ plus $5 \mu \mathrm{g}$ of streptavidin) and incubated at ambient temperature for $4 \mathrm{~h}$. The beads were washed three times in

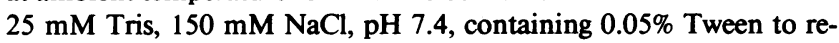
move unbound AP5. The chymotryptic digest of IIb-IIIa, to which was added $1 \mathrm{mM}$ PMSF, $2 \mathrm{mM}$ EDTA and $3 \mathrm{mM}$ EGTA, was mixed with the AP5-streptavidin such that the molar ratio between AP5 and the major chymotryptic fragment of IIIa was estimated to be $1: 1$. The mixture was incubated at $4^{\circ} \mathrm{C}$ overnight with agitation and then transferred to a disposable polypropylene column (Econo-column; Bio-Rad Laboratories). Unbound material was collected, and the column was washed with three column volumes of Tris-saline buffer, and one column volume of distilled water. Finally, bound proteins were eluted using three column volumes of $100 \mathrm{mM}$ glycine, $\mathrm{pH} 2.2$. The eluate was immediately neutralized by collecting it into a pretitrated volume of $1 \mathrm{~N}$ $\mathrm{NaOH}$, dialyzed against $5 \mathrm{mM}$ Tris, $\mathrm{pH} 6.8$, containing $0.1 \mathrm{mM}$ PMSF, and evaporated to dryness (Speed-Vac; Savant Instruments, Hicksville, NY) or concentrated using a Micro-Pro-Dicon unit $(10,000 \mathrm{~mol} w \mathrm{wt}$ cutoff; Bio-Molecular Dynamics, Beaverton, OR). Microsequencing of protein fragments was performed for a fee by the Protein Sequencing Facility of the Medical College of Wisconsin, Milwaukee, WI, using a protein sequencer (No. 477A; Applied Biosystems, Inc., Foster City, CA).

\section{Results}

Initial characterization of the human autoantibody $R A$. The specificity of RA for IIb-IIIa was first established by modified antigen capture ELISA (MACE) using murine MAb, AP2, to capture IIb-IIIa from platelet lysates presensitized with autoantibodies. RA also reacted with IIb-IIIa in the ACE (9), wherein solid-phase AP2 was used to capture IIb-IIIa from platelet lysates before reaction with primary antibody. Immunoblotting of platelet lysates prepared from the patient's platelets as well as platelets of $\mathrm{PI}^{\mathrm{A} 1}$ or $\mathrm{Pl}^{\mathrm{A} 2}$ homozygous donors confirmed that $\mathrm{RA}$ antibody binds to a protein migrating as IIIa. The binding of RA antibody is independent of $\mathrm{Pl}^{\mathrm{A}}$ or Pen polymorphisms which are both expressed on IIIa $(9,25)$.

Autoreactive epitopes were further characterized using purified IIb-IIIa in an immunoblot assay (Fig. 1). In addition to a prominent IIIa band with an apparent molecular mass of 93 $\mathrm{kD}$, high molecular mass bands at $\sim 210$ and $165 \mathrm{kD}$ that likely represent dimers and multimers of IIIa (26) were bound
Figure 1. Immunoblot assay to determine the specificity of RA antiserum. Purified IIb-IIIa was

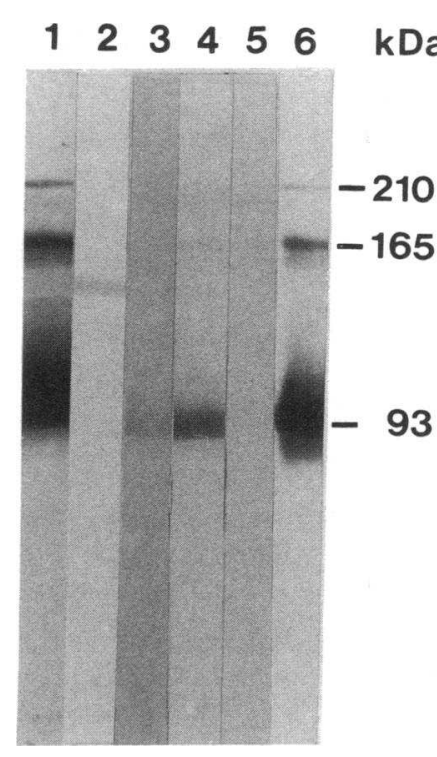
solubilized in SDS and separated under nonreduced conditions by SDS-PAGE using $7 \%$ polyacrylamide resolving slab gel. The separated proteins were electrotransferred to nitrocellulose. The following antibodies were tested: RA antiserum (lane 1); murine MAb AP4 (lane 2), which was used as a marker for IIb; SD, a second human autoantiserum recognizing IIIa (lane 3); anti$\mathrm{Pl}^{\wedge 2}$ (lane 4); human blood group AB control serum (lane 5); and murine MAb AP5 (lane 6). The positions of IIIa (93 $\mathrm{kD})$ and two protein bands related to IIIa (165 and $210 \mathrm{kD}$ ) are indicated to the right of the gel.

by RA (lane 1) and AP5 (lane 6). Also shown are the binding of a second autoantibody, SD (lane 3) and anti-PI ${ }^{\mathrm{A} 2}$ (lane 4). IIb protein was located by the binding of AP4 (lane 2). Negative control serum is depicted in lane 5 .

By flow cytometry, RA IgG autoantibodies bind to intact platelets (data not shown). No difference in binding was observed when nonactivated and thrombin-activated platelets were compared. RA IgG eluted from immunoblots was able to bind to intact platelets in flow cytometry, and RA IgG eluted from intact platelets bound to IIIa in the immunoblot assay.

Autoreactive epitopes on proteolytic fragments of purified $I I b-I I I a$. The conditions for extensive digestion of IIb-IIIa with chymotrypsin were established in order to obtain the smallest fragment of IIIa still reactive with RA. The results of digestion with chymotrypsin for $16-20 \mathrm{~h}$ at $37^{\circ} \mathrm{C}$, using a protease to substrate ratio of 1:7, are depicted in Fig. 2. By immunoblot assay, under nonreduced conditions, the ability of chymotryptic fragments to be bound by polyclonal rabbit anti-IIb and anti-IIIa, as well as autoantibody RA and MAb AP5 was tested. The starting material contained intact IIb but also IIb fragments with apparent molecular masses of 66,41 , and $35 \mathrm{kD}$ (lane $A$ ). However, the majority of IIIa was intact, only a small amount of a $65-\mathrm{kD}$ fragment detectable (lane $C$ ). After digestion, the only remnant of IIb detectable was a trace fragment at $27 \mathrm{kD}$ (lane $B$ ). Thus glycoprotein IIb had been completely digested by this procedure. On the other hand, fragments of IIIa corresponding to $65,50,38$, and $33 \mathrm{kD}$ were detected (lane $D$ ), the predominant fragment being that at $50 \mathrm{kD}$. Autoantibody RA binds to intact IIIa (lane $E$ ) and the three IIIa fragments with apparent molecular masses under nonreduced conditions of 50,38 , and $33 \mathrm{kD}$ (lane $F$ ). AP5 reacts with intact IIIa (lane $G$ ) and the predominant 50-kD IIIa fragment (lane $H$ ). Upon reduction of disulfide bonds, the reactivity of the 50-kD fragment with both RA and AP5 was lost (data not shown). To further characterize the chymotryptic fragments, they were separated under nonreduced conditions and subsequently reduced within the same polyacrylamide gel before transfer to nitrocellulose membrane (Fig. 3). Rabbit anti- 

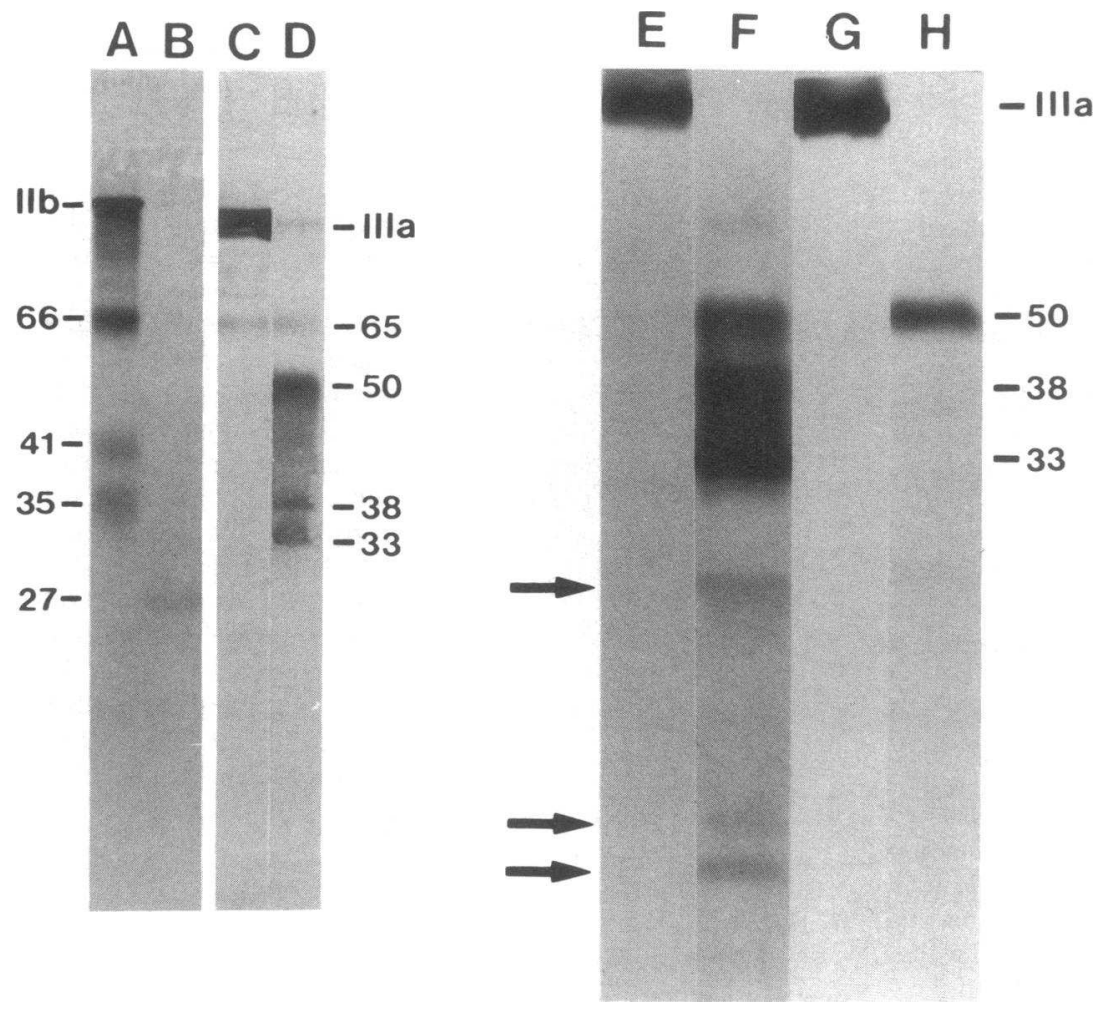

Figure 2. Effect of chymotrypsin digestion of purified IIb-IIIa on expression of the RA and AP5 epitopes as determined by an immunoblot assay. IIb and IIIa fragments were separated by SDSPAGE using a $12.5 \%$ polyacrylamide resolving gel under nonreduced conditions. Intact IIb-IIIa (lanes $A, C, E, G$ ) and the chymotryptic digest (lanes $B, D, F$, and $H$ ) were analyzed. Lanes $A$ and $B$ were incubated with rabbit polyclonal anti-IIb; lanes $C$ and $D$, with rabbit polyclonal anti-IIIa; lanes $E$ and $F$, with autoantibody RA; and lanes $G$ and $H$, with AP5. The three protein bands in lane $F$ with apparent molecular masses lower than $33 \mathrm{kD}$ (arrows) represent chymotrypsin itself. The latter reaction is an artifact of the immunoblot assay wherein certain secondary antibodies bind nonspecifically to chymotrypsin. The locations of bands corresponding to IIb and IIIa are indicated. Numbers correspond to the apparent molecular mass of indicated bands in kilodaltons.

IIIa $_{636-654}$ recognizes chymotryptic fragments with apparent molecular masses from 33 to $50 \mathrm{kD}$ (lane 2). Rabbit antiIIIa $_{1-13}$ against the amino-terminus of IIIa binds only to the 50-kD fragment (lane 4). The binding to intact IIIa of antiIIIa $_{636-654}$ and anti-IIIa ${ }_{1-13}$ is also shown (lanes 1 and 3, respectively). From these data, one can conclude that the autoantibody RA binds to a chymotryptic fragment of IIIa as small as $33 \mathrm{kD}$, which does not carry the amino-terminus of the molecule, but does contain the sequence 636-654.

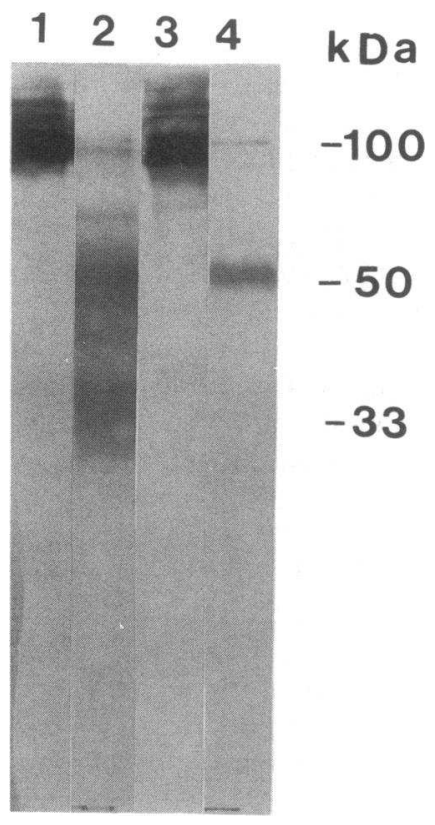

The chymotryptic 50-kD core fragment of IIIa. The 50-kD chymotryptic core fragment was purified by immunoaffinity chromatography using a streptavidin-agarose matrix to capture biotinylated AP5. The antigen composition of the purified 50$\mathrm{kD}$ fragment was then analyzed by immunoblot assay (Fig. 4).

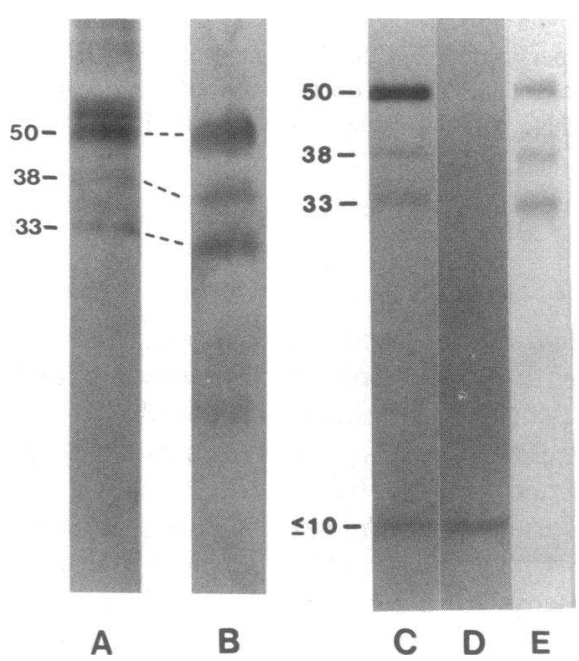

Figure 4. Composition of the affinity-purified $50-\mathrm{kD}$ fragment as determined by immunoblot assay with autoantibody RA (lane $A$ ), rabbit polyclonal anti-IIIa $a_{636-654}$ (lanes $B$ and $E$ ), polyclonal rabbit antibody against IIIa ${ }_{1-13}$ (lane $D$ ) and by a silver stain of an SDS-PAGE gel (lane $C$ ). The positions of protein bands with apparent molecular masses of $50,38,33$, and $\leq 10 \mathrm{kD}$ are indicated. The $50-\mathrm{kD}$ core fragment was subjected to electrophoresis under nonreduced conditions in lanes $A$ and $B$, but under reduced conditions in lanes $C-E$. 
Under nonreduced conditions, autoantibody RA bound to protein bands at 50,38 , and $33 \mathrm{kD}$ (lane $A$ ). Two less prominent bands $>50 \mathrm{kD}$ were also resolved. These may represent minor analogues of the $50-\mathrm{kD}$ fragment that are produced by chymotryptic cleavage at positions more amino-terminal or more carboxy-terminal to the termini of the predominant 50 $\mathrm{kD}$ fragment. However, a more likely explanation is that these $>50-\mathrm{kD}$ bands represent fractions of the $50-\mathrm{kD}$ band that have undergone spontaneous partial reduction and therefore migrate with a slightly decreased electrophoretic mobility. These retain reactivity with $\mathrm{RA}$ as they are not completely reduced. Rabbit anti-IIIa ${ }_{636-654}$ also bound to protein bands with apparent molecular masses similar to the 50,38 , and $33 \mathrm{kD}$ fragments (lane $B$ ). The $50-\mathrm{kD}$ sample analyzed in lane $\mathrm{B}$ was obtained on a different occasion than that in lane $A$ and does not exhibit partially reduced $50-\mathrm{kD}$ bands. Under reduced conditions, the silver staining of the purified $50-\mathrm{kD}$ fragment confirmed the presence of protein bands with apparent molecular masses of 33,38 , and $50 \mathrm{kD}$ fragments (lane $C$ ). The aminoterminus of the reduced $50-\mathrm{kD}$ fragment is residue 427 , based upon the microsequence IVQVTFD. The amino-terminus of the reduced 33-kD fragment, the smallest fragment reactive with RA autoantibody, is residue 479 , based upon a microsequence of XPSQQDEXSP. An amino-terminal peptide (molecular mass $\leq 10 \mathrm{kD}$ ) was detected by both silver stain (lane $C$ ) and rabbit anti-IIIa ${ }_{1-13}$ (lane $D$ ). Each of the reduced 33, 38,

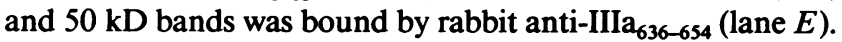
These data suggest that the " 50 " $\mathrm{kD}$ fragment seen on nonreduced gels actually represents a composite of disulfide-bonded fragments. In conclusion, RA reacts only with nonreduced chymotryptic fragments of IIIa that bind anti-IIIa ${ }_{636-654}$. We find no evidence that the amino-terminus of IIIa is required for autoantibody reactivity. In fact, the smallest fragment reacting with autoantibody, the $33-\mathrm{kD}$ fragment, does not carry the amino-terminal epitope recognized by anti-IIIa $a_{1-13}$.

Additional results. In ACE using AP5, the nonreduced 50$\mathrm{kD}$ fragment was bound by both RA and anti-PI ${ }^{\mathrm{Al}}$ antibodies. Thus, the AP5 epitope is distinct from sites recognized by anti$\mathrm{PI}^{\mathrm{A} 1}$ or RA antiserum because the binding of AP5 did not inhibit the binding of the latter antibodies.

By direct antigen ELISA, purified IIb-IIIa or the AP5-affinity purified $50-\mathrm{kD}$ fragment were used to screen for additional autoantibodies against IIb-IIIa that were not detectable by the immunoblot assay. Sera from 131 patients with suspected autoimmune thrombocytopenia were assayed. Clinical follow-up was obtained on 70 patients such that they could be categorized as having: primary ITP (39 patients), secondary ITP (6 patients), drug-induced immune thrombocytopenia ( 7 patients), or nonimmune thrombocytopenia (18 patients) (see Table I). Although the binding of human autoantibodies is normally weaker than the binding of anti-P1 ${ }^{\mathrm{Al}}$ or AP5, we detected significantly positive reactions with sera from 33 patients (Tables I and II). These 33 patients included individuals with each of four clinical types of thrombocytopenia. The distribution of clinical types of thrombocytopenia within the 33 patients was not different from that of the total group of 70 patients for whom a clinical follow-up was obtained. Positive reactions were considered to be those which exceeded the mean plus 3 SD of the values obtained with nonreactive patient antisera ( $n$ $=51$ ).

Of 39 patients with primary ITP, 17 (43.6\%) had serum antibodies that bound to IIb-IIIa or the $50-\mathrm{kD}$ fragment. The majority of these patients had chronic ITP, where 15 of 31 patients (48.4\%) expressed serum antibodies of this specificity. In eight patients with acute ITP, two were found to have antibodies that bound to the $50-\mathrm{kD}$ fragment but not to IIb-IIIa. Interestingly, antibodies specific for IIb-IIIa or the $50-\mathrm{kD}$ fragment were also found in one case of ITP secondary to lymphoma, one case of HIV-associated ITP, one case of drug-induced thrombocytopenia and 6 of 18 cases (33.3\%) of apparent nonimmune thrombocytopenia. It must be pointed out, however, that in a number of these cases, coincidence of primary or secondary ITP could neither be established nor excluded. As seen in Table II, the two patients whose sera gave the strongest positive reactions in the ELISA did not have ITP; the former presented with chronic liver disease and alcoholism; the latter appears to have a mild form of the Bernard-Soulier syndrome. 20 sera reacted with the $50-\mathrm{kD}$ fragment but bound marginally, if at all, to intact IIb-IIIa. Of 13 sera (including RA) that were positive for IIb-IIIa, 12 also bound to the $50-\mathrm{kD}$ fragment.

These data confirm that the $50-\mathrm{kD}$ fragment contains autoantigens frequently encountered in autoimmune thrombocytopenia but also indicate that such antibodies are not restricted to ITP. The data also demonstrate that two classes of antigens exist: those like RA that are expressed on intact IIIa as well as the fragment; and a second group that may represent neoantigens that are cryptic on the intact IIIa molecule.

\section{Discussion}

There is increasing evidence of the importance of IIb-IIIa as a carrier of autoantigens in chronic ITP. As many as one-half of

Table I. Patients with Suspected Immune Thrombocytopenia

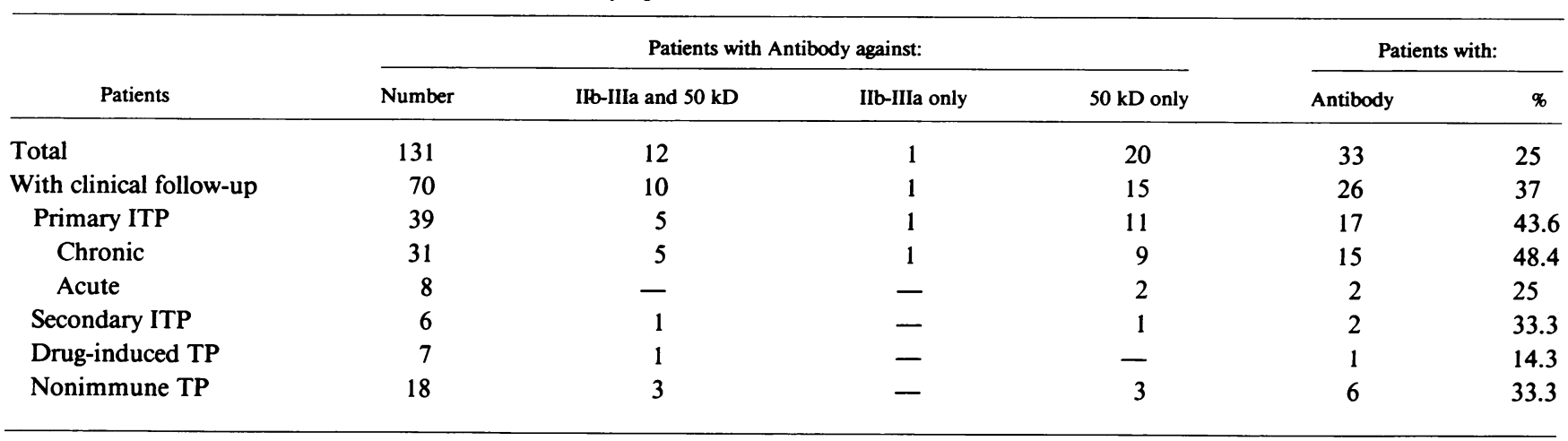


Table II. Reaction of Human Antisera with Purified 50-kD Fragment or Intact IIb-IIIa as Determined by ELISA

\begin{tabular}{|c|c|c|c|c|c|c|c|}
\hline Patient & $\mathrm{Dx}^{*}$ & $50 \mathrm{kD}$ & IIb-IIla & Patient & Dx & $50 \mathrm{kD}$ & IIb-IIIa \\
\hline 1 & $\mathbf{N}$ & $\underline{0.322^{\ddagger}}$ & $\underline{0.102}$ & 18 & - & $\underline{0.110}$ & 0.054 \\
\hline 2 & $\mathbf{N}$ & 0.235 & $\overline{0.027}$ & 19 & - & $\overline{0.103}$ & $\underline{0.117}$ \\
\hline 3 & C & 0.213 & 0.044 & 20 & $\mathbf{N}$ & $\underline{0.100}$ & 0.072 \\
\hline 4 & $\mathbf{N}$ & $\underline{0.204}$ & $\underline{0.189}$ & 21 & $\mathrm{C}$ & $\underline{0.097}$ & 0.040 \\
\hline 5 & $\mathrm{C}$ & 0.193 & 0.071 & 22 & $\mathrm{~N}$ & 0.097 & 0.050 \\
\hline 6 & A & $\underline{0.159}$ & $\overline{0.043}$ & 23 & $\mathrm{C}$ & $\underline{0.096}$ & 0.052 \\
\hline 7 & C & 0.148 & 0.109 & 24 & C & 0.093 & 0.055 \\
\hline 8 & - & $\underline{0.147}$ & 0.051 & 25 & $\mathrm{C}$ & $\underline{0.093}$ & 0.027 \\
\hline 9 & $\mathbf{S}$ & 0.144 & 0.069 & 26 & - & 0.093 & 0.031 \\
\hline 10 & $\mathrm{C}$ & $\underline{0.139}$ & $\overline{0.053}$ & 27 & $\mathrm{C}$ & $\underline{0.091}$ & 0.036 \\
\hline (RA) 11 & $\mathrm{C}$ & $\underline{0.136}$ & $\underline{0.171}$ & 28 & D & $\underline{0.090}$ & $\underline{0.077}$ \\
\hline 12 & C & $\underline{0.131}$ & $\underline{0.077}$ & 29 & $\mathrm{C}$ & $\underline{0.089}$ & 0.027 \\
\hline 13 & A & $\underline{0.129}$ & 0.064 & 30 & - & $\underline{0.089}$ & 0.027 \\
\hline 14 & - & $\underline{0.129}$ & $\underline{0.126}$ & 31 & $\mathbf{N}$ & $\underline{0.086}$ & 0.027 \\
\hline 15 & - & $\overline{0.124}$ & $\overline{0.027}$ & 32 & $\mathrm{C}$ & $\overline{0.084}$ & 0.052 \\
\hline 16 & $\mathbf{S}$ & $\underline{0.121}$ & 0.063 & 33 & $\mathrm{C}$ & 0.035 & $\underline{0.129}$ \\
\hline 17 & $\mathrm{C}$ & $\underline{0.116}$ & 0.046 & & & & \\
\hline & & & & \multicolumn{2}{|c|}{$50 \mathrm{kD}$} & \multicolumn{2}{|c|}{ IIb-IIIa } \\
\hline \multicolumn{4}{|c|}{ Negative Patients (mean $\pm S D ; n=51$ ) } & \multicolumn{2}{|c|}{$0.033 \pm 0.014$} & \multicolumn{2}{|c|}{$0.027 \pm 0.013$} \\
\hline \multicolumn{4}{|c|}{ Normal Subjects $(n=7)$} & \multicolumn{2}{|c|}{$0.028 \pm 0.030$} & \multicolumn{2}{|c|}{$0.026 \pm 0.019$} \\
\hline \multicolumn{4}{|c|}{ Positive Controls: Anti-PI ${ }^{\mathbf{A I}}$} & \multicolumn{2}{|c|}{0.602} & \multicolumn{2}{|c|}{0.755} \\
\hline \multicolumn{4}{|c|}{ AP5 } & \multicolumn{2}{|c|}{0.801} & \multicolumn{2}{|c|}{0.646} \\
\hline
\end{tabular}

Abbreviations: * Dx, Diagnosis; $\mathrm{N}$, nonimmune thrombocytopenia; C, chronic ITP; A, acute ITP; S, secondary autoimmune thrombocytopenia; D, drug-induced immune thrombocytopenia.

‡ Absorbance at $405 \mathrm{~nm}$; average of duplicate determinations. Values that are underlined are greater than $3 \mathrm{SD}$ above the mean obtained with negative patient samples and are thus considered to represent positive reactions.

patients with chronic ITP may have circulating autoantibodies specific for platelet membrane glycoprotein IIb-IIIa $(14,16)$. A study of the binding characteristics of certain autoantibodies against IIb-IIIa, based upon their ability to compete with the binding of four murine monoclonal antibodies, argues that the number of autoantigenic epitopes is not small (27). However, competitive binding between human autoantisera and murine MAbs can only give a general impression of the number of and relationship between autoepitopes. But, heterogeneity of autoantigens on IIb-IIIa is also reflected in the finding that certain, albeit a minority of autoantibodies induce platelet dysfunction without causing destruction $(28,29)$, while the majority are associated with significant thrombocytopenia.

None of the epitopes recognized by human autoantisera reactive with IIb-IIIa have been precisely identified. One human monoclonal autoantibody against IIIa, 5E5 (30) binds predominantly to activated or altered platelets suggesting that the epitope is cryptic. Tomiyama et al. (12) have characterized two autoantisera against the IIb heavy chain that react with a chymotryptic $65 \mathrm{kD}$ fragment (reduced molecular mass) retained by platelets after proteolysis. The only autoantigenic epitope that has been precisely identified is that recognized by another human monoclonal autoantibody, 2E7 (31). 2E7 binds to an epitope defined by the sequence $231-238$ of IIb that is destroyed by proteolysis with chymotrypsin (31).
In this study, we present a more systematic approach to the characterization of prototypical autoantigenic epitopes on IIIa. We selected the immunoblot assay, which had been successfully applied to this problem earlier by Beardsley et al. (11), as one of the initial screening procedures. This approach defined an epitope(s) that is recognized by a particularly strong autoantiserum, the RA prototype, which is only expressed by nonreduced IIIa. Although RA antiserum was initially detected by its strong reactivity with denatured IIIa, it has consistently reacted with intact platelets, platelet membranes or purified IIb-IIIa in all of the assays used in this study.

To further localize the RA epitope(s) on IIIa, we used the immunoblot assay to assess the binding of RA autoantibodies to electrophoretically separated chymotryptic fragments of IIIa. Our aim was to define the smallest fragment retaining the RA epitope(s). Results obtained with RA were compared with those obtained with the murine MAb AP5 and human anti$\mathrm{Pl}^{\mathrm{A} 1}$. Earlier, this approach led to the localization of the $\mathrm{Pl}^{\mathrm{Al}}$ epitope to a 66-kD chymotryptic fragment of IIIa (32) and the Bak $^{\mathrm{a}}$ epitope to the carboxy-terminal portion of the IIb heavy chain (6). The RA epitope is expressed by a number of chymotryptic fragments, ranging from 33 to $50 \mathrm{kDa}$, whereas AP5 and anti-PI ${ }^{\mathrm{A} 1}$ only react with the $50-\mathrm{kD}$ species. Polyclonal rabbit antibodies against synthetic peptides derived from the IIIa sequence were used to localize these fragments. Rabbit anti-IIIa $\mathrm{A35}_{6554}$ crossreacts with the same series of fragments (33-50 kD), separated under nonreducing conditions, and only the $50-\mathrm{kD}$ fragment carried the amino-terminus of IIIa, as evidenced by its reaction with rabbit anti-IIIa $a_{1-13}$. These results suggested that the autoantigenic epitope $R A$ resides in the carboxy-terminal region of the $50-\mathrm{kD}$ fragment. When this $50-\mathrm{kD}$ fragment is affinity purified and reduced, it itself generates fragments with apparent molecular masses of $\leq 10,33,38$, and $50 \mathrm{kD}$.

A logical explanation of these data is diagramed in Fig. 5 which depicts the probable relationship of the $\leq 10,33$ and 50 $\mathrm{kD}$ fragments. Digestion of the cysteine-rich region of IIIa must be limited to a handful of sites generating a limited number of fragments that are still held together by disulfide bonds. The largest fragment of $50 \mathrm{kD}$ (under reduced conditions), which

$$
\leq 10 \mathrm{kDa} \quad 50 \mathrm{kDa}
$$

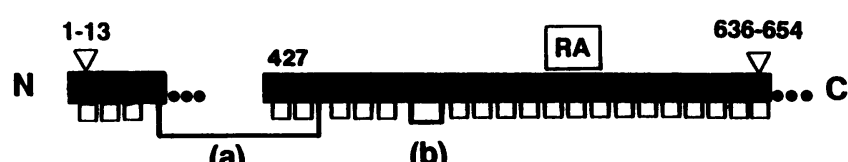

(a)

(b)

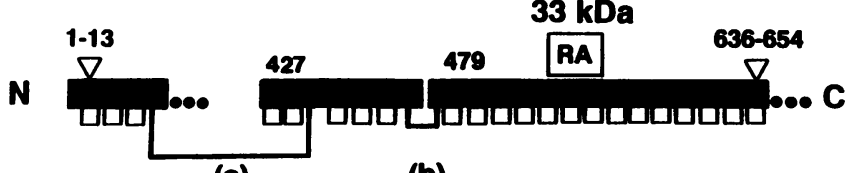

(a)

(b)

Figure 5. Proposed relationship of the $\leq 10,33$, and $50 \mathrm{kD}$ chymotryptic fragments of IIIa. Brackets represent disulfide bonds; bond a bridges the amino-terminal $\leq 10-\mathrm{kD}$ fragment with the carboxy-terminal 50-kD fragment that begins at residue 427 ; bond $b$ spans the second chymotrypsin cleavage site between tyrosine- 478 and arginine-479. Epitopes recognized by rabbit polyclonal antibodies are in dicated $(\nabla)$ as is the general location of the prototype RA autoantigen epitope(s). Dotted lines indicate that the precise carboxy-termini of the $\leq 10,33$, and $50 \mathrm{kD}$ fragments remain to be determined. 
extends from residue 427 to at least residue 654 , can be digested further into fragments of 33 or $38 \mathrm{kD}$, both of which share the same carboxy-terminal sequence that includes residues 635 to 654 . The corresponding amino-terminal fragments have not been positively identified, and these may be further digested to unidentifiable smaller fragments once the initial chymotryptic cleavage is made. The amino-terminus of the 33-kD fragment, the smallest that retains the RA epitope(s), is at position 479 of IIIa. We can thereby conclude that it consists of roughly 170 amino acids, the remaining mass contributed by carbohydrate. Regardless of the degree of chymotryptic digestion, both the 33- and the 50-kD fragments can remain disulfide-bonded internally and with the $\leq 10-\mathrm{kD}$ amino-terminal fragment of IIIa, thereby generating the nonreduced $50-\mathrm{kD}$ fragment which expresses the RA, AP5, and $\mathrm{Pl}^{\mathrm{Al}}$ epitopes. The fact that the apparent molecular mass of the nonreduced 50$\mathrm{kD}$ fragment is smaller than the sum of the reduced fragments is a result of the high degree of intrachain disulfide bonding, a phenomenon mirrored by the native IIIa molecule itself. Indeed, the entire $50-\mathrm{kD}$ fragment contains 24 disulfides, at least one of which (bond a in Fig. 5) joins the amino-terminus of IIIa to the reduced $50-\mathrm{kD}$ fragment. The fact that small amounts of the immunoreactive fragments of 33 and $38 \mathrm{kD}$ are also found before treatment with reducing agents indicates that spontaneous reduction of disulfide bond a probably occurs during generation, purification, and/or characterization of the $50-\mathrm{kD}$ fragment (Figs. 2 and 3). A subsequent hydrolysis by chymotrypsin at Tyrosine-478 generates the $33-\mathrm{kD}$ immunoreactive fragment that remains attached via a disulfide bond most likely joining cysteines 473 and 486 (bond $b$ in Fig. 5). Spontaneous reduction of disulfide bond $b$ accounts for the small amounts of 33-kD fragments detected under apparently nonreduced conditions (Figs. 2 and 3). At this time, we do not have sufficient sequence information to precisely localize the $38-\mathrm{kD}$ fragment, although, by virtue of its reactivity with the rabbit antiIIIa ${ }_{636-654}$ antibody, the 38-kD fragment probably begins somewhere between residues 428 and 479 and includes the entirety of the $33-\mathrm{kD}$ fragment.

Although the amino acid sequence of IIIa has been published, little information is available concerning the higher order structure of IIIa. It has been demonstrated that chymotryptic digestion of IIIa within the membrane of platelets produces a dipeptide consisting of a $20-\mathrm{kD}$ amino-terminal fragment disulfide-bonded to a transmembrane $65-\mathrm{kD}$ fragment that represents the carboxy-terminal half of the IIIa molecule $(33,34)$. The amino terminus of this $65-\mathrm{kD}$ fragment is at residue 348 (33). Our experimental conditions favor a more extensive proteolysis of IIIa thereby producing a truncated form of the above $65-\mathrm{kD}$ fragment.

The hypothetical conformational features of the IIIa sequence encompassing the 33-kD chymotryptic fragment (residues 420-654) can be analyzed using algorithms, based upon primary amino acid sequence, that predict secondary structure (35), positions of beta turns (36), chain flexibility (37), hydrophilicity (38) and the hydropathic index (39). Using these analyses, the segment of IIIa on or about the amino-terminus of the 33-kD fragment at arginine- 479 can be described as: $(a)$ a region that immediately follows a beta turn position at or about proline-464; $(b)$ the region of greatest flexibility (B[norm] $=1.13)$; and $(c)$ a point of high hydrophilicity $(\mathrm{Ah}=1.88)$ and lowest hydropathy (h.i. $=-25$ ). Taken together, these results would predict that arginine- 479 is located in an extended and exposed position within the $50-\mathrm{kD}$ fragment, a finding that is consistent with its experimentally determined susceptibility to further cleavage by chymotrypsin.

The affinity-purified $50-\mathrm{kD}$ fragment was used as solidphase antigen to detect other autoantibodies reactive with this fragment. By this approach, we have a preliminary indication of the frequency of antibodies recognizing epitopes within the $50-\mathrm{kD}$ fragment in patients with suspected autoimmune thrombocytopenia. From a group of 31 patients with chronic ITP, 15 sera were positive. This represents about one-half of patients with chronic ITP, a finding that conforms very well to previous estimates $(13,14)$. In addition, two of eight patients with acute ITP had antibodies that bound to the 50-kD fragment. The finding of anti-IIb/IIIa antibodies in acute ITP, albeit at lesser frequencies, is also consistent with a prior report (16). 5 of the 17 sera, including RA itself, also bound significantly to intact IIIa in the ELISA. Only one serum was reactive with IIIa but negative against the $50-\mathrm{kD}$ fragment. Consequently, among autoantigens associated with IIIa, a majority $(16 / 17)$ can be localized to the $50-\mathrm{kD}$ region.

Some autoantigens, such as the RA epitope, are defined by a site or sites on the $50-\mathrm{kD}$ fragment that are also available on intact IIIa. Other autoantigens associated with the $50-\mathrm{kD}$ fragment, apparently a majority in this case, must represent neoantigens or cryptic structures that are relatively inaccessible on intact IIIa. From our results, autoantibodies specific for the 50-kD fragment are not restricted to primary ITP and cannot be considered a hallmark of the adult, chronic form of ITP. Further studies are required to determine the role of such autoantibodies in the etiology of autoimmunity to platelets.

Of the 26 patients with antibody for whom follow-up history is available, $17(65 \%)$ had clinical histories compatible with autoimmune thrombocytopenia (either primary or secondary). The remaining nine appeared to have other causes of thrombocytopenia. Nonetheless, the additional presence of immune mechanisms of platelet destruction in the latter nine patients cannot be excluded.

The identification of regions on IIb-IIIa that are autoimmunogenic in man provides opportunities to develop new diagnostic and therapeutic approaches to autoimmune thrombocytopenia. In this report, we have taken the first concrete steps toward identifying such autoantigenic regions. Further studies are being pursued to more precisely determine the number and kinds of these epitopes in the cysteine-rich 50-kD fragment derived from IIIa.

\section{Acknowledgments}

We thank Ms. Janis Smiltneck for her excellent technical assistance.

This study was supported by NHLBI grant HL-37471 and NHLBI program grant HL-44612 awarded to T. J. Kunicki and by NHLBI grant HL-35207 awarded to J. McFarland. Dr. Kekomaki was a Senior Visiting Scientist of the Lynde and Harry Bradley Foundation at the time of this study.

\section{References}

1. Nurden, A., and J. Caen. 1974. An abnormal platelet glycoprotein pattern in three cases of Glanzmann's thrombasthenia. Br. J. Haematol. 28:253-260.

2. Phillips, D., and P. P. Agin. 1977. Platelet membrane defects in Glanzmann's thrombasthenia. Evidence for decreased amounts of two major glycoproteins. J. Clin. Invest. 60:535-545.

3. Kunicki, T. J., and R. H. Aster. 1978. Deletion of the platelet specific alloantigen $\mathrm{PI}^{\wedge}$ from platelets in Glanzmann's thrombasthenia. J. Clin. Invest. 61:1225-1231 
4. van Leeuwen, E., A. von dem Borne, L. von Riesz, L. Nijenhuis, and C. Engelfriet. 1981. Absence of platelet-specific alloantigens in Glanzmann's thrombasthenia. Blood. 57:49-54.

5. Boizard, B., and J.-L. Wautier. 1984. Lek ${ }^{\star}$, a new platelet antigen absent in Glanzmann's thrombasthenia. Vox Sang. 46:47-54.

6. Kieffer, N., B. Boizard, D. Didry, J.-L. Wautier, and A. T. Nurden. 1984 Immunochemical characterization of the platelet-specific alloantigen Lek: a comparative study with the $\mathrm{PI}^{\wedge}$ alloantigen. Blood. 64:1212-1219.

7. von dem Borne, A., E. von Riesz, F. Verheugt, J. ten Cate, J. Koppe, C Engelfriet, and L. Nijenhuis. 1980. Bak², a new platelet-specific antigen involved in neonatal alloimmune thrombocytopenia. Vox Sang. 39:113-120.

8. Shibata, Y., T. Miyaji, Y. Ichikawa, and I. Matsuda. 1986. A new platelet antigen system Yuk $^{\mathrm{a}} / \mathrm{Yuk}^{\mathrm{b}}$. Vox Sang. 51:334-336.

9. Furihata, K., D. J. Nugent, A. Bissonette, R. H. Aster, and T. J. Kunicki 1987. On the association of the platelet-specific alloantigen, Pen², with glycoprotein IIIa. J. Clin. Invest. 80:1624-1630.

10. van Leeuwen, E. F., J. Th. M. van der Ven, C. P. Engelfriet, and A. E. G. $\mathrm{Kr}$. von dem Borne. 1982. Specificity of autoantibodies in autoimmune thrombocytopenia. Blood. 59:23-26.

11. Beardsley, D. S., J. E. Spiegel, M. M. Jacobs, R. I. Handin, and S. E. Lux 1984. Platelet membrane glycoprotein IIIa contains target antigens that bind anti-platelet antibodies in immune thrombocytopenias. J. Clin. Invest. 74:17011707.

12. Tomiyama, Y., Y. Kurata, Y. Shibata, S. Honda, T. Furubayashi, H. H Mizutani, T. Tsubakio, T. Yonezawa, and S. Tarui. 1989. Immunochemical characterization of an autoantigen on platelet glycoprotein Ilb in chronic ITP. comparison with the Bake alloantigen. Br. J. Haematol. 71:77-83.

13. Woods, V. L., E. H. Oh, D. Mason, and R. McMillan. 1984. Autoantibodies against the platelet glycoprotein IIb/III a complex in patients with chronic ITP. Blood. 63:368-375.

14. McMillan, R., P. Tani, F. Millard, P. Berchtold, L. Renshaw, and V. L. Woods. 1987. Platelet-associated and plasma anti-glycoprotein autoantibodies in chronic ITP. Blood. 70:1040-1045.

15. Kiefel, V., S. Santoso, M. Weisheit, and C. Mueller-Eckhardt. 1987. Monoclonal antibody-specific immobilization of platelet antigens (MAIPA): a new tool for the identification of platelet-reactive antibodies. Blood. 70:1722-1726.

16. Berchtold, P., R. McMillan, P. Tani, S. Sommerville-Nielsen, and V. S. Blanchette. 1989. Autoantibodies against platelet membrane glycoproteins in children with acute and chronic immune thrombocytopenic purpura. Blood. 74:1600-1602.

17. Kunicki, T., and R. Aster. 1983. Qualitative and quantitative tests for platelet alloantibodies and drug-dependent antibodies. In Methods in Hematology: Immune Cytopenias. R. McMillan, editor. Churchill-Livingstone, New York. 49-67.

18. Pidard, D., R. R. Montgomery, J. S. Bennett, and T. J. Kunicki. 1983. Interaction of AP2, a monoclonal antibody specific for the human platelet glycoprotein IIb-IIla complex, with intact platelets. J. Biol. Chem. 258:12582-12586.

19. Fitzgerald, L. A., B. Steiner, S. C. Rall Jr., S.-S. Lo, and D. R. Phillips. 1987. Protein sequence of endothelial glycoprotein IIIa derived from a cDNA clone. Identity with platelet glycoprotein IIIa and similarity to "integrin." J. Biol. Chem. 262:3936-3939.

20. George, J. N. 1978. Studies on platelet plasma membranes. IV. Quantitative analysis of platelet membrane glycoproteins by $\left({ }^{125} \mathrm{I}\right)$-diazotized diiodosulfanilic acid labeling and SDS-polyacrylamide gel electrophoresis. J. Lab. Clin. Med. 92:430-446.

21. Furihata, K., J. Hunter, R. H. Aster, G. R. Koewing, N. R. Shulman, and
T. J. Kunicki. 1988. Human anti-P $\mathrm{I}^{\mathrm{E} 1}$ antibody recognizes epitopes associated with the alpha subunit of platelet glycoprotein Ib. Br. J. Haematol. 68:103-110.

22. Markwell, M. A. K., S. M. Haas, L. L. Bieber, and N. E. Tolbert. 1978. A modification of the Lowry procedure to simplify protein determination on membrane and lipoprotein samples. Anal. Biochem. 78:206-210.

23. Fitzgerald, L. A., B. Leung, and D. R. Phillips. 1985. A method of purifying the platelet membrane GPIIb-IIIa complex. Anal. Biochem. 151:169-174.

24. Kunicki, T. J., P. J. Newman, D. L. Amrani, and M. W. Mosesson. 1985. Human platelet fibrinogen: purification and hemostatic properties. Blood. 66:808-815.

25. Kunicki, T. J., and R. H. Aster. 1979. Isolation and immunologic characterization of the human platelet alloantigen $\mathrm{Pl}^{\wedge 1}$. Mol. Immunol. 16:353-360.

26. Calvete, J. J., J. L. McGregor, G. Rivas, and J. Gonzales-Rodriguez. 1987. Identification of a glycoprotein IIIa dimer in polyacrylamide gel separations of human platelet membranes. Thromb. Haemostasis. 58:694-697.

27. Tsubakio, T., P. Tani, V. L. Woods, and R. McMillan. 1987. Autoantibodies against platelet GPIIb/IIIa in chronic ITP react with different epitopes. Br. J. Haematol. 67:345-348.

28. Niessner, H., K. J. Clemetson, S. Panzer, C. Mueller-Eckhardt, S. Santoso, and P. Bettelheim. 1986. Acquired thrombasthenia due to GPIIb/IIIa-specific platelet autoantibodies. Blood. 68:571-576.

29. Balduini, C., G. Grignani, F. Sinigaglia, A. Bisio, L. Pacchiarini, D. Rota S. Calabring, C. Balduini, C. Mauri, and E. Ascari. 1987. Severe platelet dysfunction in a patient with autoantibodies against membrane glycoproteins IIb/IIIa. Hemostasis. 1:98-104.

30. Nugent, D. J., T. J. Kunicki, C. Berglund, and I. D. Bernstein. 1987. A human monoclonal autoantibody recognizes a neoantigen on glycoprotein IIIa expressed on stored and activated platelets. Blood. 70:16-22.

31. Kunicki, T. J., E. F. Plow, R. Kekomaki, and D. J. Nugent. 1990. Human monoclonal autoantibody $2 \mathrm{E} 7$ is specific for a peptide sequence of platelet glycoprotein IIb. Localization of the epitope to $\mathrm{IIb}_{231-238}$ with an immunodominant Trp $_{235}$. J. Autoimmun. In press.

32. Kornecki, E., S.-Y. Chung, J. C. Holt, C. S. Cierniewski, G. P. Tuszynski, and S. Niewiarowski. 1985. Identification of $\mathrm{Pl}^{\wedge}$ alloantigen domain on a $66 \mathrm{kDa}$ protein derived from glycoprotein IIIa of human platelets. Biochim. Biophys. Acta 818:285-290.

33. Niewiarowski, S., K. J. Norton, A. Eckardt, H. Lukasiewicz, J. C. Holt, and E. Kornecki. 1989. Structural and functional characterization of major platelet membrane components derived by limited proteolysis of glycoprotein IIIa. Biochim. Biophys. Acta 983:91-99.

34. Beer, J., and B. S. Coller. 1990. Evidence that platelet glycoprotein IIIa has a large disulfide-bonded loop that is susceptible to proteolytic cleavage. J. Biol. Chem. 264:17564-17573.

35. Garnier, J., D. J. Osguthorpe, and B. Robson. 1978. Analysis of the accuracy and implications of simple methods for predicting the secondary structure of globular proteins. J. Mol. Biol. 120:97-120.

36. Chou, P. Y., and G. D. Fasman. 1979. Prediction of beta-turns. Biophys. J. 26:367-373.

37. Karplus, P. A., and G. E. Schulz. 1985. Prediction of chain flexibility in proteins: a tool for the selection of peptide antigens. Naturwissenschaften. 72:212-213.

38. Hopp, T. P., and K. R. Woods. 1981. Prediction of protein antigenic determinants from amino acid sequences. Pro. Natl. Acad. Sci. USA. 78:38243828.

39. Kyte, J., and R. F. Doolittle. 1982. A simple method for displaying the hydropathic character of a protein. J. Mol. Biol. 157:105-132. 